\title{
Proposal of fruit battery method for estimating oil palm ripeness
}

\begin{abstract}
This paper presents fruit battery evaluation as a method to distinguish between oil palm fruit ripeness stages. The fruit battery method consists of two electrodes that have different ionization tendencies. The fruit battery can detect oil palm fruit electrolyte changes from decreasing moisture content as the oil palm ripens. This paper proposes a condition to distinguish oil palm ripeness by varying the depth and interval between electrodes. Subsequently, the probability of estimating oil palm fruit moisture content using this method is evaluated using a regression analysis.
\end{abstract}

Keyword: Fruit battery; Oil palm; Regression analysis; Electrolyte 